BULL. AUSTRAL. MATH. SOC.

VOL. $3(1970), 231-264$.

\title{
Some product varieties of groups
}

\section{R. A. Bryce and John Cossey}

We consider varieties $\underline{\underline{\mathrm{V}}}=\underline{\underline{\mathrm{A}}} \underline{p} \underset{\mathrm{A}}{\mathrm{A}} \stackrel{\mathrm{A}}{\mathrm{p}}$ with $m$ prime to $p$. We show that the subvariety lattice of $\underline{V}$ is distributive and has descending chain condition and that $\stackrel{A}{A}=p$ is its only just non-Cross subvariety. When $m$ is prime we determine the join-irreducible subvarieties of $\mathrm{V}$. The method involves fairly detailed description of the structure of non-nilpotent critical groups in $\mathrm{V}$.

\section{Introduction}

The principal motivation behind many investigations in the theory of varieties of groups since its inception seems to have been a desire to decide whether or not every variety requires only finitely many laws to define it; and a large number of varieties do have this property (which is usuaily known as 'the finite basis property'). The papers [1], [3], [6], [11], [17], [21], for example, all contain finite basis theorems. There have been conjectures that every variety has the finite basis property and, more cautiously, that every soluble-of-finite-exponent variety does. Recently, however, two (unpublished) counter-examples to this have been produced: the first, by A.Yu. Ol'shanskii, is soluble of length 5 and exponent 120 and the second, by M.R. Vaughan-Lee, is soluble of length 4 and exponent 16 . One of the results proved here (Theorem 5.1) goes a small way towards closing the gap between these examples and known finitely based varieties of smaller soluble length.

Even before these examples of 01 'shanski and Vaughan-Lee were known the range of questions considered, for locally finite varieties at any

Received 16 June 1970. 
rate, had widened considerably as people found that, from methods developed to prove finite basis theorems, much more information could be obtained; see, for example, [1], [2], [4, 5], [7], [15], [16]. The test questions, on which one can determine the efficacy of one's methods for dealing with a given variety $\underline{\underline{V}}$ then, include those following.

a) Does $\underline{\underline{\mathrm{V}}}$, and all its subvarieties, have the finite basis property?

b) Is the lattice $\Lambda(\underline{\underline{\mathrm{V}}})$ of subvarieties of $\underline{\underline{\mathrm{V}}}$ distributive? If the answer to (a) is 'yes' for $\underline{\underline{V}}$ then every subvariety of $\underline{\underline{V}}$ can be written as a finite join of (finitely) join-irreducible subvarieties.

c) What are the join-irreducible subvarieties of $\mathrm{V}$ ?

If $\underline{\underline{v}}$ is not a Cross variety it has subvarieties which are just non-Cross (Kovács and Newman [16]).

d) What are the just non-Cross subvarieties of $\underline{v}$ ?

This list is far from exhaustive, of course - we have not, for example, mentioned Graham Higman's interesting question about the orders of the free groups of $\underline{\underline{V}},(\xi 2$ in [12]) - but it is with these questions in mind that the present paper has been written. The varieties $\underline{V}$ with which we will be concerned are $\underset{p}{A} \stackrel{A}{\Rightarrow} \underset{m}{A}$ where $p$ is a prime not dividing $m$; we answer (a), (b) affirmatively and provide answers to (c), (d). The reader is referred to Hanna Neumann [18] for definitions and terminology about varieties of groups and to Curtis and Reiner [8] for representation theory.

The technique employed involves fairly detailed description of the structure of non-nilpotent critical groups in $\underline{\underline{V}}$, and may be regarded as a natural development of the methods of Chapter 3 in [5] (see also [4]): in particular the concepts of bigroup and variety of bigroups used there will be needed here. The structure theorems are proved in $\S 4$, while other preliminary results which will be needed in $\$ 5$ are introduced in $\$ \$ 2,3$; $\$ 2$ deals with representations of groups in $\underset{\Rightarrow}{A} \stackrel{A}{A}$ over fields of characteristic $p$ and 53 with enough representation theory over the ring of integers modulo $p^{\alpha}$ for our present purposes. 
A convention used needs comment. If $A$ is an abelian normal subgroup of a group $G$ we shall often regard $A$ as a $G$-module and may, without comment, write $A$ additively. The action of elements of $G$ on A will be written $a^{g}(a \in A, g \in G)$, but note that other linear transformations of $A$ may be written as right multiplication; thus if $e$ is an endormorphism of the module $A$ we write ae for the image of $a$ under $e$.

\section{Representations of $A_{n} A_{p}$ groups}

For convenience we start by stating a well-known theorem in a form appropriate for our purposes (see Higman [10, Lemma]).

LEMMA 2.1. Let $A$ be an abelian p-group, $K$ a finite group of automorphisms of $A$ and $K_{1}$ a normal $p^{\prime}$-subgroup of $K$. If $A_{0}$ is the subgroup of $A$ whose elements are fixed by every element of $K_{1}$ then $A_{0}$ has a complement in $A$ which admits $K$.

This section is devoted to proving the following theorem.

THEOREM 2.2. Let $p$ be a prime and $m$ a natural number prime to $p$, and let $K$ in $\underset{\Rightarrow}{A} \stackrel{A}{\Rightarrow}$ be a finite group which has a faithful irreducible representation over a field $E$ of characteristic $p$. All the faithful irreducible representation modules for $K$ over $E$ are principal indecomposables and the representations they afford form a single linear isonorphism class.

Proof. Start by assuming that $E$ is algebraically closed and let $M$ be a faithful co-monolithic module for $K$ over $E$, with unique maximal submodule $M_{0}$, say. Write $S$ for the normal Hall $p^{\prime}$-subgroup of $K$ : notice that $S$ is not 1 . By Maschke's Theorem there exists an irreducible submodule $N$ of $M_{S}$ outside $M_{0} \cdot$ Now $N$ is one dimensional and, since $S$ is normal in $K, N k$ is a submodule of $M_{S}$ whenever $k \in K$. Hence if $T$ is a transversal of $K$ to $S$

$$
M=\sum_{t \in T} N t
$$


Suppose $k \in K$ is such that $N \cong N k$. That is, there is a one-to-one linear transformation $\theta: N \rightarrow N k$ such that

$$
(n s) \theta=(n \theta) s, s \in S, n \in N \text {. }
$$

However $s$ acts simply as a scalar multiplication, say $n s=n \alpha(s)$ where $\alpha(s) \in E$; so that if $n \theta=n^{\prime} k$, then $n \mapsto n^{\prime}$ is a linear transformation and

$$
(n s)^{\prime}=(n \alpha(s))^{\prime}=n^{\prime} \alpha(s)=n^{\prime} s, n \in N, s \in S .
$$

We conclude from (2.4) and (2.5) that

$$
\left(n^{\prime} k\right) s=(n s)^{\prime} k=\left(n^{\prime} s\right) k, n^{\prime} \in N, s \in S,
$$

whence it follows that $[S, k] \leq \operatorname{ker} N$. Now $S$ centralizes $K^{\prime}$ and therefore $[S, k]$ is normal in $K$. Lemma 2.1, and the fact that $M$ is faithful and indecomposable, ensures that $[S, k]=1$; but $c_{K}(S)=S$ or else $K$ would have a non-trivial normal $p$-subgroup and could not have a faithful irreducible representation over $E$. Hence $k \in S$ and it follows from (2.3) that

$$
M=\underset{t \in T}{\bigoplus} N t
$$

We have shown, therefore, that the dimension of $M$ is $|K: S|$. A simple application of Lemma 2.1 and Maschke's Theorem shows that $M / M_{0}$ is faithful and, being irreducible, is co-monolithic, so that

$$
\operatorname{dim} M=\operatorname{dim} M / M_{0} ;
$$

in other words $M_{0}=0$. The first statement of the theorem is now proved (for closed fields) by observing that a principal indecomposable for $K$ over $E$ is co-monolithic and that it is faithful if the co-monolith is faithful.

The restriction on the field can now be removed. If $V$ is a faithful irreducible module for $K$ over $E$, and $E^{*}$ is the closure of $E$ then, by (70.15) of Curtis and Reiner [8], $E^{*} \bigotimes_{E} V$ is completely reducible and each irreducible component is faithful, hence projective, by what has already been proved; and therefore $E^{*} \Theta_{E} V$ is projective.

This in turn will imply that $V$ is projective. For, if 


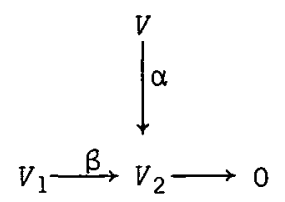

is a diagram with exact row then there exists $\gamma^{*}$ such that the diagram

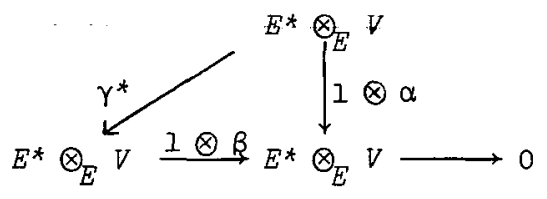

commutes. If $v \in V$ and $(1 \otimes v) \gamma^{*}=\sum e_{i} \otimes v_{i}$ (where $\left\{1=e_{0}, e_{1}, \ldots\right\}$ is a basis for $E^{*}$ over $E$ ) it is easily checked that $\gamma: v \mapsto v_{1}$ is an $E K$-homomorphism such that $\gamma \beta=\alpha$, as required. Finally then, $V$ is a direct sum of principal indecomposables and is therefore itself a principal indecomposable.

To proceed further, more structure on $K$ is required. The lemma that follows comes either directly from, or by routine modification of, results of Kochendörffer [13] and Taunt [20].

LEMMA 2.7. $S$ is a direct product of indecomposable normal homocylic subgroups $s_{i}(1 \leq i \leq r)$ of $K$. Moreover each $\sigma S_{i}$ is a minimal normal subgroup of $K$ and $o K$ is their direct product, this being the unique decomposition of $\sigma K$ as a direct product of minimal normal subgroups of $K$.

In order to prove that the faithful irreducibles of $K$ over $E$ form a single linear isomorphism class it suffices, by Theorem 2.5 in [1], to assume that $E$ is algebraically closed. If, then, $M$ is a faithful irreducible module for $K$ over $E$ (2.6), and (44.1) in [8], ensures that

$$
M \cong N^{K}
$$

where $N$ is a one-dimensional submodule of $M_{S}$. The proof consists in choosing a basis for $M$ and a set of generators for $K$, depending on $M$, and evaluating the matrices representing these generators; it will be obvious that the linear group they generate is independent of $M$. 
In (2.6) $T$ may be chosen as a complement for $S$ and is to be fixed throughout. Also the subgroups $S_{i}(1 \leq i \leq r)$ in Lemma 2.7 are to be fixed. Regard $\sigma S_{i}$ and $S_{i} / \Phi\left(S_{i}\right)$ as $T$-modules over a field of prime order (NOTE: $S_{i}$ has prime-power order); they are easily seen to be isomorphic and hence, by 12.2 .2 of [9], $\operatorname{ker\sigma } S_{i}=C_{T}\left(S_{i}\right)$. With at most one exception (by Lemma 2.7 at most one $\sigma S_{i}$ is central in $K$ ) $\left|T: C_{T}\left(S_{i}\right)\right|$ is therefore equal to $p ;$ choose a fixed $t_{i} \in T \backslash C_{T}\left(S_{i}\right)$. Next, given the faithful irreducible module $M$ choose $N$ so that (2.8) holds; let $L=\operatorname{ker} N$. Note that $S / L$ is cyclic, hence $S_{i} / S_{i} \Omega$ are all cyclic and that, because of Lemmas 2.7 and 2.1 ,

$$
\left|S_{i} / S_{i} \cap L\right|=\exp S_{i}, \quad|S / L|=\exp S, \quad 1 \leq i \leq r .
$$

Choose an element $s \in S \backslash L$ whose order is $\exp S$.

The following lemma is vital.

LEMMA 2.10. Let $V$ be a free module of rank $\rho$ over the ring of integers moduzo $q^{\alpha}$ ( $q$ a prime), and let $V_{0}$ ( $\$ q V$ ) be a free submodule of $V$ of rank $\rho-1 . H=\langle h\rangle$ is a $q^{\prime}$-cycle acting faithfulzy and indecomposably on $V$. There is a basis $\left\{v_{1}, \ldots, v_{p}\right\}$ of $V$ such that $v_{i} \in V_{0}(1 \leq i \leq p-1)$ and

$$
v_{i} h=v_{i+1}, \quad 1 \leq i \leq \rho-1 .
$$

Moreover the matrices representing $h$ with respect to all bases with the property (2.11) are the same.

Proof. No proper submodule of $V$, not in $q V$, admits $h$. It follows easily that if

$$
U_{i}=V_{0} \cap V_{0} h \cap \ldots \cap V_{0} h^{i-1}, 1 \leq i \leq \rho,
$$

then the rank of $U_{i}$ is $\rho-i$. Choose $0 \neq v_{\rho-1} \in U_{\rho-1}$. Since $U_{\rho-1}=U_{\rho-2} \cap U_{\rho-2} h$ there exists $v_{\rho-2} \in U_{\rho-2}$ such that $v_{\rho-1}=v_{\rho-2}{ }^{h}$ and $v_{\rho-2} \notin U_{\rho-1}$. In a similar fashion choose $v_{\rho-3}, \ldots, v_{1}\left(\epsilon v_{0}\right)$ 
and define $v_{\rho}=v_{\rho-1} h$. Now $\left\{v_{1}, \ldots, v_{\rho-1}\right\}$ is a basis for $v_{0}$ by construction and hence $v_{\rho} \notin v_{0}$ so that $\left\{v_{1}, \ldots, v_{\rho}\right\}$ is a basis for $V$, and has the desired properties. Notice that the coefficients in the expression for $v_{\rho} h$ are coefficients of the minimum polynomial for $h$. This completes the proof of Lemma 2.10.

Choose a basis $\left\{v_{i 1}, \ldots, v_{i p(i)}\right\}$ for $s_{i}$ respecting $S_{i} \cap L$ as in the lerma, with $t_{i}$ playing the rôle of $h$ : for convenience set $s_{i}=v_{i \rho(i)}$. Then there exists an integer $m_{i}$, independent of $L$, such that

$$
s^{m_{i}} \equiv s_{i}^{u_{i}} \bmod L
$$

where $\left(u_{i}, \exp S_{i}\right)=1$. However $\left\{v_{i l}^{u_{i}}, \ldots, v_{i p(i)}^{u_{i}}\right\}$ is still a basis for $S_{i}$ with the properties of Lemma 2.10 , so, without loss of generality,

$$
s^{m_{i}} \equiv s_{i} \bmod L \text {. }
$$

Now $s_{i}$ is a generator of $S_{i}$ as T-module and the action of $T$ on $s_{i}$ depends only on that of $t_{i}$; specifically, to each $t \in T$ there exists an integer $\tau(i, t)$, independent of $L$ by Lemma 2.10, such that

$$
s_{i}^{t} \equiv s_{i}^{\tau(i, t)} \bmod L .
$$

Finally let $N=\operatorname{sp}\{n\}, n s=\alpha n(\alpha \in E)$, and $\underline{\underline{B}}=\{n \otimes t: t \in T\}$ be our basis for $M$ (from (2.8) this is possible). Note that

$$
(n \otimes t) s_{i}=n \otimes t s_{i}=n \otimes s_{i}^{t^{-1}} t=n s_{i}^{\tau\left(i, t^{-1}\right)} \otimes t=\alpha^{m_{i} \tau\left(i, t^{-1}\right)}(n \otimes t) .
$$

If $U$ is the representation afforded by $M$ then, with respect to $\underline{\underline{B}}$, $s_{i} U$ has diagonal matrix, and the diagonal entries are powers of $\alpha$. Our choice of the quantities $m_{i}, \tau(i, t)$ was independent of $M$. Hence if $U^{\prime}$ is a faithful irreducible representation of $K$ and $\alpha^{\prime}$ is the analogue of $\alpha$, then $\alpha^{\prime}$ is a power of $\alpha$ and so, with respect to a 
suitable basis, $s_{i}^{\prime} U^{\prime}$ is a power of $s_{i} U$. Since the matrix of $t U$ with respect to $\underline{B}$ is a permutation matrix for all $t \in T$ we see that the linear group $K U$ is independent of the faithful irreducible $U$.

\section{Further preliminaries}

Let $p$ be a prime and $R_{\alpha}$ the ring of integers modulo $p^{\alpha}$. We need some facts about representations over $R_{\alpha}$, analogues of well known facts about representations over fields.

If $K$ is a finite group the group ring $R_{\alpha} K$ has minimum condition on right ideals. Let $R_{\alpha} K=\bigoplus_{i=1}^{\rho} A_{i}$ be a decomposition of $R_{\alpha} K$ as a direct sum of indecomposable right ideals. Since $R_{\alpha} K / p R_{\alpha} K$ and $p^{\alpha-1} R_{\alpha} K$ are isomorphic vector spaces over $R_{1}$ it follows in a familiar fashion that each $A_{i}$ is a free $R_{\alpha}$-module. By (54.11) in [8] each $A_{i} / p A_{i}$ is a principal indecomposable of $R_{1} K$.

Next suppose that $N_{1}$ is an irreducible module for $K$ over $R_{1}$, and write $E_{1}=\operatorname{End}_{K} N_{1}$, a finite field isomorphic to $\mathrm{GF}\left(p^{d}\right)$, say. Let $C_{1}$ be the multiplicative group of $E_{1}$; then $N_{1}$ is a $C_{1} K$-module over $R_{1}$ and we shall show that if $N_{1}$ is projective as $R_{1} K$-module it is projective as $R_{1} C_{1} K$-module. Choose a fixed isomorphism $\varphi: G F\left(p^{d}\right) \rightarrow E_{1}$ thus turning $N_{1}$ into a $G F\left(p^{d}\right) K$-module - call it $\hat{N}_{1}$. Now $\hat{N}_{1}$ is projective since it occurs as a direct summand of $G \mathrm{~F}\left(p^{d}\right) \otimes_{R_{1}} N_{1}$, and $N_{1}$ is projective (using somewhat more than 70.15 of [8]; L.G. Kovács (unpublished) has proved our assertion). If $c \in C_{1}$ there exists $e \in G F\left(p^{d}\right)$ for which, in $\hat{N}_{1}$,

$$
n c=n e, n \in N_{1} ;
$$

and using this and the projectivity of $\hat{N}_{1}$ one easily checks the commativity of the diagrams which ensure that $N_{1}$, as $R_{1} C_{1} K$-module, is projective. 
The last two paragraphs are now brought together. Let $N_{\alpha} \quad\left(\alpha \in I^{+}\right)$ be homocyclic groups of exponent $p^{\alpha}$, say with $N_{\beta}=N_{\alpha} / p^{\beta} N_{\alpha} \quad(\alpha \geq \beta)$; $E_{\alpha}$ is the endomorphism ring, and $A_{\alpha}$ the automorphism group, of $N_{\alpha}$. Define $\mu_{\alpha \beta}: E_{\alpha} \rightarrow E_{\beta}$ by

$$
\left(x+p^{\beta} N_{\alpha}\right)\left(e \mu_{\alpha \beta}\right)=x e+p^{\beta} N_{\alpha}, x \in N_{\alpha}, e \in E_{\alpha} .
$$

It is easy to see that $\mu_{\alpha \beta}$ is an onto ring homomorphism such that $\mu_{\alpha \beta} \mu_{\beta \gamma}=\mu_{\alpha \gamma}(\alpha \geq \beta \geq \gamma)$, and that the restriction $\nu_{\alpha \beta}$ of $\mu_{\alpha \beta}$ to $A_{\alpha}$ as multiplicative homomorphism is onto $A_{B}$. Suppose now that $K_{1}$ is a subgroup of $A_{1}$ such that $N_{1}$, as $R_{1} K_{1}$-module, is irreducible and principal indecomposable. If, as above, $E_{1}=\operatorname{End}_{K_{1}} N_{1}$ and $C_{1}$ is its multiplicative group then $N_{1}$ as $R_{1} C_{1} K_{1}$-module is principal indecomposable and hence there are subgroups $C_{\alpha}, K_{\alpha}$ of $A_{\alpha}$, which centralize each other, such that $\nu_{\alpha 1}$ takes $C_{\alpha}$ isomorphically onto $C_{1}$ and $K_{\alpha}$ isomorphically onto $K_{1}$. We may assume also that $K_{\alpha} \nu_{\alpha \beta}=K_{B}$, $C_{\alpha \nu_{\alpha \beta}}=C_{\beta}$. In this set up we have

LEMMA 3.1. As $R_{\alpha} K_{\alpha}$-module $N_{\alpha}$ has the double centralizer property. Also if $E_{\alpha}=$ End $_{K_{\alpha}} N_{\alpha}$ then every element $e$ of $E_{\alpha}$ can be written uniquely as

$$
e=\sum_{i=0}^{\alpha-1} p^{i} c_{i}, \quad c_{i} \in C_{\alpha} \cup\{0\} .
$$

Proof. The double centralizer property is proved first, by induction on $\alpha$; for $\alpha=1$ it is true by $(26.4)$ in [8]. We show in fact that if $\xi \in E_{\alpha}$ and $\xi$ centralizes $c_{\alpha}$ then $\xi=\sum r_{i} k_{i}$ for some $r_{i} \in R_{\alpha}$ and $k_{i} \in K_{\alpha}$.

$$
\text { Notice that } \operatorname{ker} \mu_{\alpha \alpha-1}=p^{\alpha-1} E_{\alpha} \text { and that } \xi \mu_{\alpha \alpha-1} \text { centralizes } c_{\alpha-1}
$$
and so, by induction, there exists $r_{i}^{\prime} \in R_{\alpha}, k_{i}^{\prime} \in K_{\alpha}$ so that 


$$
\xi=\sum r_{i}^{\prime} k_{i}^{\prime}+p^{\alpha-1} e
$$

for some $e \in E_{\alpha}$. Since $\lambda: x+p N_{\alpha} \rightarrow p^{\alpha-1} x$ is a group isomorphism of $N_{1}$ onto $p^{\alpha-1} N_{\alpha}$ and, from (3.2), $p^{\alpha-1} e$ centralizes $C_{\alpha}$, for all $c \in C_{\alpha}$ and all $x \in N_{\alpha}$ we have

$$
\begin{aligned}
\left(x+p N_{\alpha}\right)\left((e c) \mu_{\alpha 1}\right) \lambda=\left(x e c+p N_{\alpha}\right) \lambda & =p^{\alpha-1}(x e c) \\
= & x\left(p^{\alpha-1} e\right) c=x c\left(p^{\alpha-1} e\right)=\left(x+p N_{\alpha}\right)\left((c e) \mu_{\alpha 1}\right) \lambda .
\end{aligned}
$$

Hence $(e c) \mu_{\alpha I}=(c e) \mu_{\alpha I}$. Therefore $e \mu_{\alpha l}$ centralizes $C_{1}$. As $\operatorname{ker} \mu_{\alpha \perp}=p E_{\alpha}$ there exists $e^{\prime} \in E_{\alpha}, r_{i}^{\prime \prime} \in R_{\alpha}$ and $k_{i}^{\prime \prime} \in K_{\alpha}$ such that

$$
e=\sum r_{i}^{\prime \prime} k_{i}^{\prime \prime}+p e^{\prime}
$$

Combining this with (3.2) yields the desired result. The remainder of Lemma 3.1 can be proved by entirely similar methods.

It is well-known that there exists a linear transformation $\beta_{1}$ of $N_{1}$ such that $B_{1}^{-1} e B_{1}=e^{p} \quad\left(e \in E_{1}\right):$ as $E_{1}$-space $N_{1}$ is completely reducible and, on each irreducible component of $N_{1}, e$ and $e^{p}$ have the same minimal polynomial and the matrix of each is similar to the companion matrix of this minimum polynomial. More generally we have

LEMMA 3.3. There exists $B_{\alpha} \in E_{\alpha}$ such that

$$
\beta_{\alpha}^{-1} c \beta_{\alpha}=c^{p}, \quad c \in C_{\alpha} \text {. }
$$

The proof of this will follow from the next lemma and the fact that $\operatorname{kerv}_{\alpha 1}$ is a p-group $(12.2 .2$ in [9]).

LEMMA 3.4. Two $p^{\prime}$-elements of a finite group are conjugate if and only if they are conjugate modulo a normal p-subgroup.

Proof. Suppose that $X$ is a finite group, $Y$ is a normal p-subgroup of $X$ and $x_{1}, x_{2}$ are conjugate modulo $Y$. It suffices to assume $y$ is abelian. For each $t \in Y h$ there exists $y_{t} \in Y$ such that 


$$
x_{1}^{t}=x_{2} y_{t}
$$

We show that for some $t, y_{t} \in C$, the centralizer of $x_{2}$ in $Y$. For, suppose $t, u$ are such that $y_{t} y^{-1} \in C$; then $x_{1}^{t} x_{1}^{-u} \in C$ and so $\left[u^{-1} t, x_{1}^{-u}\right] \in C$. Now $C$ has a complement $y_{1}$ in $y$ by Lemma 2.1, which admits $x_{2}$. Write $u^{-1} t=c y_{1} \quad\left(c \in C, y_{1} \in Y_{1}\right)$ and then

$$
\left[c y_{1}, x_{1}^{-u}\right]=\left[c y_{1}, y_{u}^{-1} x_{2}^{-1}\right]=\left[y_{1}, x_{2}^{-1}\right] \in Y_{1} \cap c=1 \text {, }
$$

whence $y_{1}=1$. Therefore $u^{-1} t \in C$. It follows that, if $T$ is a transversal of $y$ to $C,\left\{y_{t}: t \in h^{r}\right\}$ is also a transversal of $y$ to $C$, and hence for some $t, y_{t}$ centralizes $x_{2}$ as we asserted above. For this $t,\left(x_{1}^{t}\right)^{n}=x_{2}^{n} y_{t}^{n}$ which, if $n$ is the 1.c.m. of the ( $\left.p^{\prime}-\right)$ orders of $x_{1}, x_{2}$, gives $y_{t}^{n}=1$ whence $y_{t}=1$, completing the proof.

LEMNiA 3.5. Two faithful principal indecomposable $R_{\alpha}$ K-modules $N_{\alpha}$ and $P_{\alpha}$ afford linearly isomorphic representations if and only if $N_{\alpha} / p N_{\alpha}$ and $P_{\alpha} / p P_{\alpha}$ afford (faithful) linearly isomorphic representations of $R_{1} K$.

Proof. The 'only if' direction is easy, so suppose that $N_{\alpha}$ and $P_{\alpha}$ afford representations $T, U$ respectively and that the representations $T^{\prime}, U^{\prime}$ thereby induced on $N_{\alpha} / p N_{\alpha}$ and $P_{\alpha} / p P_{\alpha}$ are linearly isomorphic. That is, with respect to suitably chosen bases, the matrix groups $K T^{\prime}$ and $K U^{\prime}$ are equal. Hence there is an automorphism $\lambda$ of $K$ such that

$$
k \lambda T^{\prime}=k U^{\prime}, k \in K \text {. }
$$

The module $N_{\alpha} / P N_{\alpha}$ affording the representation $\lambda T^{\prime}$ is therefore isomorphic to $P_{\alpha} / P P_{\alpha}$. Hence by (54.14) in [8], and the remarks at the beginning of this section, $N_{\alpha}$ as module affording the representation $\lambda T$ is isomorphic to $P_{\alpha}$. In particular, with respect to suitably chosen 
bases, the matrix groups $K \lambda T$ and $K U$ are equal. Hence $T, U$ are linearly isomorphic.

Suppose that $V_{1}, V_{2}$ are irreducible modules affording faithful representations $T_{1}, T_{2}$ of a group $K$. One can form the groups $X_{1}, X_{2}$ by split-extending $V_{1}, V_{2}$ by $K$ with actions $T_{1}, T_{2}$ respectively. It is easy to check that $T_{1}$ is linearly isomorphic to $T_{2}$ if and only if $X_{1}$ and $X_{2}$ are isomorphic groups. In view of this we have the following corollary to Lemma 3.5 .

LEMMA 3.6. If $K$ is an irreducible linear group acting on a space $N_{1}$ over $R_{1}$, the split-extension is a uniquely determined critical group. If, moreover, $N_{1}$ as $R_{1} K$-module is principal indecomposable then to each $\alpha \in I^{+}$there exists a unique split-extension $N_{\alpha} K$ such that $N_{\alpha} K / p N_{\alpha} \cong N_{1} K ; N_{\alpha} K$ is critical. Furthermore if $N$ is abelian of exponent $p^{\alpha}$ and an extension $N K$ exists such that $N K / p N \cong N_{1} K$ then $N$ is homocyclic and, indeed, principal indecomposable as $R_{\alpha} K$-module, and $N K \cong N_{\alpha} K$

Proof. The existence of $N_{\alpha} K$ has already been shown, and the criticality follows from (1.65) in [14] of Kovács and Newman. That $N$ is homocyclic follows from the fact that $x+p N \rightarrow p^{\alpha-1} x$ is a K-homomorphism. The remainder will be proved by Lemma 3.5 when we show that $N$ is principal indecomposable. This follows easily from the projectivity of $N_{\alpha}$.

\section{Structure of certain critical groups}

Let $G$ be a critical group the last non-trivial term of whose lower nilpotent series, $A$ say, is abelian. Then, by Theorem 3 in [10] of Higman, $A$ has a complement $B$ in $G$. Since $G$ is monolithic, $A$ is self-centralizing in $G$ and, for some prime $p, A$ is a $p$-group. We will be interested in cases when $B=H \times K$ with $H$ the maximal normal p-subgroup of $B$ and $K$ a group whose faithful irreducible representations over $G F(p)$ are projective: whenever $G$ is 
abelian-by-nilpotent of $G \in \underset{\underline{\underline{A}}}{\underline{\alpha} \underline{\underline{A}} \stackrel{A}{\Rightarrow} p}(p \nmid m)$, and $G$ is not nilpotent, this is easily seen to be the case (using Theorem 2.2 in the latter case). In this section we describe the structure of $A$ as $B$-module: in fact we show that it suffices to obtain a description of $A_{H}$ and $A_{K}$. Our aim is to construct another group (denoted later by $G^{H}$ ) which generates the same variety as $G$ but which is easier to work with then $G$ itself.

As $B$-module, then, $A$ is faithful and monolithic and, by 51.37 in [18], co-monolithic also; let $A_{0}$ be the unique maximal submodule of $A$. Write $A^{*}=p A+[A, H]$ so that $A^{*} \leq A_{0}$. Choose $h_{i j} \in z_{i}(H)-z_{i-1}(H) \quad(1 \leq i \leq c, 1 \leq j \leq r(i)-H$ has class $c$, say,) such that

$$
A(i)=\left[A, h_{11}, \ldots, h_{1 r(1)}, \ldots, h_{i 1}, \ldots, h_{i r(i)}\right]
$$

is non-trivial for each $i \in\{1, \ldots, c\}$ but that

$$
\left[A(i), z_{i}(H)\right]=1,1 \leq i \leq c \text {. }
$$

Let $p^{\delta}$ be the exponent of $A(c)$. Then it is easy to check that the mapping

$$
\xi: a+A^{*} \leftrightarrow\left[a, h_{11}, \ldots, h_{1 r(1)}, \ldots, h_{c 1}, \ldots, h_{c r(c)}\right]^{p^{\delta-1}}
$$

is a non-zero $B$-homomorphism of $A / A^{*}$. Now $\sigma A$ is in $\left(A / A^{*}\right) \xi$ and is therefore centralized by $H$; hence $K$ acts irreducibly on $\sigma A$ and, by Lemma 2.1, faithfully also. But our assumptions on $K$ mean that $(\sigma A)_{K}$ is injective so that

$$
\left(A / A^{*}\right) \xi=\sigma A \oplus U
$$

where $U$ admits $K$ and therefore $B$ since $H$ acts trivially on $\left(A / A^{*}\right) \xi$; thus $U=0$ and $\left(A / A^{*}\right) \xi=\sigma A$. It follows that $\operatorname{ker} \xi=A_{0} / A^{*}$ and that, as $B$-modules,

$$
A / A_{0} \cong \sigma A
$$

We aim now to delineate the module structure of $A$ commencing with 
the next lemma.

LEMMA 4.2. There exists a principal indecomposable submodule $N$ of $A_{K}$ such that $N / p N \cong \sigma A$ as $R_{1} K$-modules and $N$ generates $A$ as H-moduze.

Proof. Since $(\sigma A)_{K}$ is projective so is $\left(A / A_{0}\right)_{K}$ by (4.1). Hence there exists a submodule $C_{1}$ of $A_{K}$ so that

$$
C_{1}+A_{0}=A, \quad C_{1} \cap A_{0}=p A .
$$

But $C_{1} / p A \cong A / A_{0}$ so, for the same reason, there is a submodule $C_{2}$ of $C_{1}$ such that $C_{2}+p A=C_{1}, \quad C_{2} \cap p A=p C_{1}$ and $C_{2} / p C_{1} \cong A / A_{0}$. In this way construct a descending sequence of submodules $C_{i}$ such that $C_{i} / p C_{i-1} \cong A / A_{0}$. For some $j$ we must have $p C_{j}=p C_{j-1}$ which means that $C_{j} / p C_{j} \cong A / A_{0}$ is irreducible and the last assertion of Lemma 3.5 then gives that $C_{j}$ is a free $R_{\alpha}$-module. Note that $C_{i} \neq A_{0}$ for any $i$. Put $N=C_{j}$ and then, since $N$ admits $K, N$ generates $A$ as $H$-module, whence $N$ has exponent $p^{\alpha}$. This completes the proof of Lemma 4.2 .

Let the $N$ of Lemma 4.2 have $R_{\alpha}$-basis $\left\{n_{1}, \ldots, n_{s}\right\}$. Write $M_{i}$ for the $H$-submodule of $A$ generated by $n_{i} \quad(1 \leq i \leq s)$. Using von Dyck's theorem one checks that group isomorphisms $\delta_{i}: M_{1} H \rightarrow M_{i} H$ may be defined by

$$
n_{1} \delta_{i}=n_{i}, h \delta_{i}=h, h \in H
$$

The mapping

$$
(m, n) \leftrightarrow \sum_{i=1}^{s} r_{i}\left(m \delta_{i}\right), m \in M_{1}, n \in N
$$

where $n=\sum r_{i} n_{i}$, is easily seen to be balanced. Hence there exists a homomorphism $\tau$ of $M_{1} \otimes_{R_{\alpha}} N$ onto $A$ such that 


$$
(m \otimes n) \tau=\sum_{i=1}^{s} r_{i}\left(m \delta_{i}\right), m \in M_{1}, n \in N .
$$

Moreover, if $B \times K$ acts on $M_{1} \otimes_{R} N$ in the usual outer tensor product fashion:

$$
(m \otimes n)^{h k}=m^{h} \otimes n^{k}, \quad m \in M_{1}, n \in N ; h k \in H \times K,
$$

then $\tau$ is an $H \times K$-epimorphism. Define $G^{\#}$ to be the group obtained by split-extending $M_{1} \otimes_{R_{\alpha}} N\left(=A^{\#}\right)$ by $H \times K$; then $\tau$ extends to $a$ group epimorphsim $\tau: G^{\#}+G$.

Next write $E=\operatorname{End}_{K} N$. We can extend the action of $E$ to the whole of $A^{\#}$ by identifying $E$ with $1 \otimes E$ :

$$
(m \otimes n) e=m \otimes n e, m \in M_{1}, n \in N, e \in E .
$$

Then, by Lemma 3.1, $\operatorname{ker} \tau$ admits $E$. Consequently $A$ may be regarded as an $E$-space. In this set up we have:

THEOREM 4.3. If $M$ is the EH-subspace of $A$ generated by $n_{1}$ then, as $E(H \times K)$-modules,

$$
A \cong M \otimes_{E} N
$$

Proof. Since each space $p^{i} N / p^{i+1} N \quad(0 \leq i \leq \alpha-1)$ is a vector space over the field $E / p E=E_{1}, N$ has an $E$-basis $\left\{l_{1}, \ldots, z_{t}\right\}$ with $z_{1}=n_{1}$, say. Then, copying the construction of $G^{\#}$ and $\tau$, we find an $E(H \times K)$-homomorphism

$$
\nu: M \otimes_{E} N+A
$$

onto $A$. We will show that $\nu$ is one-to-one.

The construction of $v$ gives first

$$
n \in N-p N, m \in M \text { and } m \otimes n \in \operatorname{kerv} \Rightarrow m=0
$$

and second 


$$
\sigma\left(M \otimes_{E} N\right)=\sigma M \otimes_{E} N \cong \sigma M \otimes_{E_{1}} N / P N
$$

Since $\sigma M \otimes_{E} N$ is centralized by $H$ it follows from (4.4) and (4.5) that

$$
0 \neq\left(\sigma\left(M \otimes_{E} N\right)\right) \nu \leq Z(A H)=\sigma A
$$

hence $\left(\sigma\left(M \otimes_{E} N\right)\right) \nu=\sigma A$. Write $D=$ kerv $\cap \sigma\left(M \otimes_{E} N\right)$. Then, regarding $\sigma\left(M \otimes_{E} N\right)$ as an $E_{1}$-space (by (4.5)), and $\sigma A$ also, we find that the co-dimension of $D$ in $\sigma\left(M \otimes_{E} N\right)$ is precisely $\operatorname{dim} \sigma A=\operatorname{dim}_{E} N=t$. Suppose that $O M$ contains elements $m_{1}, m_{2}$ which are $E$-independent and therefore $E_{1}$-independent. Using (4.5)

$$
\operatorname{dim}\left(m_{1} \otimes N / p N+m_{2} \otimes N / p N\right)=2 \operatorname{dim} N
$$

whence, if $D \neq 0$,

$$
D_{1}=D \cap\left(m_{1} \otimes N / p N+m_{2} \otimes N / p N\right) \neq 0 .
$$

If $d \in D_{1}$ then, for suitable $x_{1}, x_{2} \in N / p N$ we have

$$
d=m_{1} \otimes x_{1}+m_{2} \otimes x_{2},
$$

and (4.4) shows that the relation $\mu: x_{1} \leftrightarrow x_{2}$ is a mapping from $X_{1}=\left\{x_{1} \in N / p N: d \in D_{1}\right\}$ to $X_{2}=\left\{x_{2} \in N / p N: d \in D_{1}\right\}$. Indeed since $D_{1} \neq 0$ and $N / p N$ is an irreducible $E_{1} K$-module, $X_{1}=X_{2}=N / p N$ and $0 \neq \mu \in E_{1}$. Hence for some non-zero $x_{1} \in N / P^{N}$,

$$
\left(m_{1}+m_{2} \mu\right) \otimes x_{1} \in D_{1}
$$

and (4.4) implies $m_{1}+m_{2} \mu=0$ contrary to the independence of $m_{1}$ and $m_{2}$. Therefore $D=0$ and hence kerv $=0$. If the $E$-dimension of $N$ is one then clearly kerv $=0$; in any case $\nu$ is one-to-one and Theorem 4.3 is proved.

The proof just completed shows that $\sigma M$ is a one dimensional $E_{1}$-space. Since $\sigma M_{1} \leq \sigma M$ we have

COROLLARY 4.6. The dimension of $\sigma M_{1}$ as $R_{1}$-space is at most the dimension $d$ of $E_{1}$ over $R_{1}$.

Write $\hat{A}=\left(M_{\perp} \otimes_{R_{\alpha}} E\right) \otimes_{E} N$ so that there is an $E$-isomorphism $\lambda: \hat{A} \mapsto A^{\#}$ which has 


$$
\lambda:\left(m_{1} \otimes 1\right) \otimes n \leftrightarrow m_{1} \otimes n, m_{1} \in M_{1}, \quad n \in N .
$$

Moreover if we define the action of $H \times K$ on $\hat{A}$ in the natural way:

$$
\left(\left(m_{1} \otimes 1\right) \otimes n\right)^{h k}=\left(m_{1}^{h} \otimes 1\right) \otimes n^{k}, m_{1} \in M_{1}, \quad n \in N,
$$

then $\lambda$ is an $H \times K$ homomorphism; and if $\hat{G}$ is obtained by extending $\hat{A}$ by $H \times K$ in this action, $\lambda$ extends to a group isomorphism $\lambda: \hat{G} \mapsto G^{\#}$. In this set up one easily checks, using Theorem 4.3

LEMMA 4.7. There exists an EH-submodule $L$ of $M_{1} \otimes_{R_{\alpha}} E$ such that $\operatorname{ker} \lambda \tau=L \otimes_{E} N$.

Next let $\beta=\beta_{\alpha}$ of Lemma 3.3 and let $\gamma: E \rightarrow E$ be defined by

$$
\gamma: e \mapsto e^{\beta}, e \in E
$$

Then it is simple to check that

$$
\lambda\left(1 \otimes \beta^{i}\right) \lambda^{-1}=\left(1 \otimes \gamma^{i}\right) \otimes \beta^{i}, \quad 0 \leq i \leq d-1 .
$$

LEMMA 4.9. $\bigcap_{i=0}^{d-1}(\operatorname{ker} \tau) \beta^{i}=0$.

Proof. It suffices to show that $\prod_{i=0}^{d-1}\left(L \otimes_{E} N\right) \lambda \beta^{i} \lambda^{-1}=0$ which, by (4.8), will follow if $\prod_{i=0}^{d-1} L\left(1 \otimes \gamma^{i}\right)=0$. Write $U=L \cap \sigma\left(M_{1} \otimes E\right)$ so that we need only prove

$$
\bigcap_{i=0}^{d-1} U\left(1 \otimes \gamma^{i}\right)=0
$$

Analogously to $(4.4)$ and $(4.5)$ we have:

$$
\begin{gathered}
m_{1} \in M_{1}, \quad e \in E-p E \text { and } m_{1} \otimes e \in L \Rightarrow m_{1}=0 ; \\
\sigma\left(M_{1} \otimes_{R_{\alpha}} E\right) \cong \sigma M_{1} \otimes_{R_{1}} E_{1} .
\end{gathered}
$$

The force of (4.12) is that to prove (4.10) we may assume that we are working in a vector space over a field $E_{1}$, where $\gamma$ is now the Galois 
automorphism $e_{1} \mapsto e_{1}^{p}$ of $E_{1}$. The following rather technical lemma will prove $(4.10)$.

LEMMA 4.13. Let $V$ be a proper non-zero submoduze of $\sigma M_{1} \otimes_{R_{1}} E_{1}$ and $l$ be maximal with respect to the property that, whenever $S$ is a linearly independent subset of $\sigma M_{1}$ containing at most $l-1$ elements, $(4.14)$

$$
0=V \cap \underset{t \in S}{\oplus} t \otimes E_{1}
$$

Then, if $S^{\prime}$ is a linearly independent subset of $\sigma M_{1}$ containing $Z$ elements

$$
0=V \cap V 1 \otimes r \cap \underset{t \in S^{\prime}}{\oplus} t \otimes E_{1}
$$

Proof. Observe first that the co-dimension of $V$ in $\sigma M_{1} \otimes E_{1}$ is at least $\cdot l-l$ and that, if $S^{\prime}$ is a linearly independent subset of $\sigma M_{1}$ containing $\tau$ elements,

$$
0 \neq V\left(S^{\prime}\right)=V \cap \underset{t \in S^{\prime}}{\oplus} t \otimes E_{1}
$$

We emulate the argument following (4.5). For all $v \in V\left(S^{\prime}\right)$ then,

$$
v=\sum_{t \in S^{\prime}} t \otimes x_{t}
$$

for some $x_{t} \in E_{1}$. By virtue of (4.14) for each $t \in S^{\prime}$ the correspondence $x_{t} \leftrightarrow x_{t^{\prime}}$ is one-to-one for each $t^{\prime} \in S^{\prime}$, and is indeed, an $E_{1}$-endomorphism of $E_{1}$. Consequently there exist elements $S^{\prime}(t)$ of $E_{1}$, linearly independent over $R_{1}$ by (4.14), such that

$$
V\left(S^{\prime}\right)=\left\{\sum_{t \in S^{\prime}} t \otimes x S^{\prime}(t): x \in E_{1}\right\} \text {. }
$$

Next define, for a linearly independent subset $S$ of $\sigma M_{1}$ containing exactly $l-l$ elements, and a basis $B$ of $O M$ containing $S$,

$$
S_{b}=S \cup\{b\}, b \in \bar{S}=B-S \text {. }
$$

Then, using (4.15), the $V\left(S_{b}\right)$ clearly generate their direct sum and each $V\left(S_{b}\right)$ has $E_{1}$-dimension $I$. Hence $\underset{b \in \bar{S}}{\oplus} V\left(S_{b}\right)$ has co-dimension $Z-1$ 
and therefore

$$
V=\underset{b \in \bar{S}}{\oplus} V\left(S_{b}\right)
$$

in particular $V$ has co-dimension exactly $\tau-1$.

We now show that for all $S$ and $B$ as above

$$
V \cap V\left(s_{b}\right) 1 \otimes \gamma=0, b \in \bar{S} \text {. }
$$

Suppose that $\omega \in V\left(S_{b}\right)$ and $w \perp \otimes \gamma \in V$. From (4.15) and (4.16)

$$
w I \otimes \gamma=\left(\sum_{t \in S_{b}} t \otimes x S_{b}(t)\right) \perp \otimes \gamma=\sum_{j \in S} \sum_{t \in S} t \otimes x_{j} S_{j}(t)
$$

for some $x, x_{j} \in E_{1}$. This implies at once that for $j \neq b, x_{j}=0$ since $j \otimes x_{j} S_{j}(j)$ occurs once only on the right and not at all on the left. Hence

$$
\sum_{t \in S_{b}} t \otimes(x \gamma) S_{b}(t)^{p}=\sum_{t \in S_{b}} t \otimes x_{b} S_{b}(t)
$$

so that

$$
(x \gamma) S_{b}(t)^{p}=x_{b} S_{b}(t), \quad t \in S_{b}
$$

If $t, t^{\prime}$ are distinct elements of $S_{b}$ (under the hypotheses $\left|S_{b}\right| \geq 2$ ) then $x_{b}\left\{S_{b}(t) S_{b}(t)^{-p}-s_{b}\left(t^{\prime}\right) S_{b}\left(t^{\prime}\right)^{-p}\right\}=0$ from which $x_{b} \neq 0$ implies $y\left\{S_{b}(t) S_{b}(t)^{-p}-S_{b}\left(t^{\prime}\right) S_{b}\left(t^{\prime}\right)^{-p}\right\}=0$ for all $y \in E_{1}$. In other words

$$
x_{b} \neq 0 \Rightarrow S_{b}(t) S_{b}(t)^{-p}-S_{b}\left(t^{\prime}\right) S_{b}\left(t^{\prime}\right)^{-p}=0 \text {, }
$$

which implies $\left(S_{b}\left(t^{\prime}\right)^{-1} S_{b}(t)\right)^{p}=S_{b}\left(t^{\prime}\right)^{-1} S_{b}(t)$, and this means that $S_{b}\left(t^{\prime}\right)^{-1} S_{b}(t) \in R_{1}$ contrary to the independence of $S_{b}\left(t^{\prime}\right), S_{b}(t)$ over $R_{1}$. We conclude that $x_{b}=0$ and therefore that $w=0$, proving (4.17). Since an arbitrary set $S^{\prime}$ of $Z$ independent elements of $\sigma M_{1}$ can always be constructed as $S^{\prime}=S_{b}$ for suitable $S$ and $B,(4.17$ ) yields 
(with $\gamma$ replaced by $\gamma^{-1}$ )

$$
V \cap V I \otimes \gamma \cap \underset{t \in S^{\prime}}{\oplus} t \otimes E_{1}=V 1 \otimes \gamma \cap V\left(S^{\prime}\right)=\left(V \cap V\left(S^{\prime}\right) 1 \otimes \gamma^{-1}\right) 1 \otimes \gamma=0
$$

and the proof of Lemma 4.13 is complete.

Return to the proof of Lenma 4.9. By (4.11) $U$ satisfies the hypotheses of Lemma 4.13 with $Z=2$. Now $U \cap U \perp \otimes \gamma$ has co-dimension at most 2 since $U, U I \otimes \gamma$ each have co-dimension 1 ; but (4.15) shows that $U \cap U 1 \otimes \gamma$ has co-dimension at least 2 , and hence exactly 2 . An easy induction using Lemma 4.13 shows that $U \cap U I \otimes \gamma \cap \ldots \cap U I \otimes \gamma^{i}$ has co-dimension $i+1$ in $\sigma M_{1} \otimes E_{1}$ and therefore, by Corollary 4.6, is zero for $i=d$. This proves (4.10) and with it Lemma 4.9 .

Lemma 4.9 provides a subdirect decomposition of $G^{\#}$ which we now describe. First note that $K^{\beta^{i}}$ centralizes $E$, and therefore, by Lemma 3.1, kert admits $K^{\beta^{i}}(0 \leq i \leq d-1)$; whence $(\operatorname{ker} \tau)^{\beta^{i}}$ admits $K$ $(0 \leq i \leq d-1)$. Consequently in $\hat{G}$,

$$
(L \otimes N) \lambda \beta^{i} \lambda^{-1}=\left(L 1 \otimes \gamma^{i}\right) \otimes N, \quad 0 \leq i \leq d-1
$$

admits $H K$. Write $M(i)=\left(M_{1} \otimes E\right) / L 1 \otimes \gamma^{i},(0 \leq i \leq d-1)$, so that $M(i)$ is an $E H$-module "Galois conjugate" to $M$. Then $G_{i}=\hat{G} /\left(L \perp \otimes \gamma^{i}\right) \otimes N$ is the group obtained by extending $M(i) \otimes_{E} N$ by $H \times K$ in the outer-tensor product fashion, and

LEMMA 4.18. $\dot{G}^{\#}$ is isomorphic to a subdirect product of $G_{0}, \ldots, G_{d-1}$.

Note that $G_{0} \cong G$. Indeed under suitable restrictions each of $G_{0}, \ldots, G_{d-1}$ is isomorphic to $G$.

LEMMA 4.19. If the faithful irreducible representations of $K$ over $G F\left(p^{d}\right)$ form a single linear isomorphism class then each of $G_{0}, \ldots, G_{d-1}$ 
is isomorphic to $G$.

Proof. If $E_{\alpha}$ is the ring in Lemma 3.1, taking $N_{1}$ to be the additive group of $\mathrm{GF}\left(p^{d}\right)$ and $K_{1}$ its multiplicative group, then Lemma 3.5 shows that the faithful indecomposable representations of $K$ over $E_{\alpha}$ are linearly isomorphic. In particular there exists $\zeta$ centralizing $E$ such that $K=K^{\beta \zeta}$. Since $\beta \zeta$ has the same action on $E$ as $B$ does we may, without loss of generality, assume $K=K^{\beta}$.

Now there exist $H$-isomorphisms $\eta_{i}: M \rightarrow M(i)$ with the property:

$$
(m e) n_{i}=\left(m m_{i}\right) e \gamma^{i}, m \in M, e \in E, i \in\{0, \ldots, d-1\},
$$

and it is easy to check that there exist isomorphisms $\theta_{i}: M \otimes_{E} N \rightarrow M(i) \bigotimes_{E} N$ with the property that

$$
(m \otimes n) \theta_{i}=m n_{i} \otimes n \beta^{i}, \quad m \in M, n \in N, i \in\{0, \ldots, d-1\} .
$$

Moreover if $a \in M \otimes_{E} N,\left(a^{h k}\right) \theta_{i}=\left(a \theta_{i}\right)^{h k^{\beta^{i}}} \quad(h \in H ., k \in K)$, and hence the mapping $\theta_{i}: G \rightarrow G_{i}$ defined by

$$
(h k a) \theta_{i}=h k^{\beta^{i}} a \theta_{i}, \quad h \in H, \quad k \in K, \quad a \in A
$$

is an isomorphsim.

COROLLARY 4.20. Under the conditions of (4.19), $\operatorname{var} G=\operatorname{var} G^{\#}$.

Proof. Use Lemmas 4.19 and 4.18 .

L.G. Kovács has constructed for us a group $K$ which has a faithful irreducible representation whose Galois conjugates, regarded as $\mathrm{GF}\left(p^{d}\right)$ representations, are not linearly isomorphic; indeed for this $k$ one can easily construct a critical group $G$ such that $G_{0}, \ldots, G_{d-1}$ are not pair-wise isomorphic. Whether or not $G$ and $G^{\#}$ nevertheless generate the same variety in general we have been unable to determine.

Summarizing this section then, we have 
THEOREM 4.21. Let $G$ be a non-nilpotent critical group the last non-trivial term of whose lower nilpotent series, $A$ say, is abelian. $A$ is a p-group, with a complement $B$ in $G$. If $B=H \times K$ where $H$ is the largest normal p-subgroup of $B$ and $K$ is such that its faithful irreducible representations over $\mathrm{GF}(p)$ are projective and form a single linear isomorphism class, then

$$
\begin{aligned}
& \operatorname{var} G=\operatorname{var} G^{\#} . \\
& \text { 5. } \underset{\mathrm{p}^{\mathrm{A}} \mathrm{am} \stackrel{\mathrm{A}}{\mathrm{p}}}{\mathrm{p}} .
\end{aligned}
$$

The first four sections can now be used to answer the test questions raised in the introduction. Much of the proof is technical in nature.

THEOREM 5.1. Every subvariety of $\underline{\underline{\mathrm{V}}}=\underline{\underline{\mathrm{A}}} \underset{p}{\mathrm{~A}} \underline{\underline{\mathrm{A}}} \mathrm{p}$ (where $p$ is a prime not dividing $m$ ) is finitely based.

THEOREM 5.2. The Zattice of subvarieties $\Lambda(\underline{\underline{V}})$ of $\underline{\underline{V}}$ is distributive.

THEOREM 5.3. The only just non-Cross subvariety of $\underline{\underline{V}}$ is $\stackrel{A}{A} p$.

The join irreducible subvarieties of $\underline{\underline{V}}$ can be described with the apparatus we have developed, but in general this is tedious without being especially illuminating. We shall content ourselves with the case when $m$ is a prime $q$. Even in this case a certain amount of preamble is necessary - it will be needed in the proof of Theorem 5.1 also.

A bigroup is a group $G$ together with an idempotent endomorphism of $G$; alternatively $G$ is a triple $(G, A, B)$ where $A$ is a normal subgroup of $G$ and $B$ a complement for $A$ in $G$. Bigroups and varieties of bigroups are discussed in $[4,5]$ and the reader is referred there for more complete information.

Let $F=\left(F, A_{1}, B_{1}\right)$ and $D=\left(D, A_{2}, B_{2}\right)$ be bigroups in which $A_{1}, A_{2}$ are abelian groups, that is $Z$-modules over the ring of integers $Z$. Let $F \# D$ be the group obtained by split-extending $A_{1} \otimes_{2} A_{2}$ by $B_{1} \times B_{2}$ with the usual outer-tensor product action: 


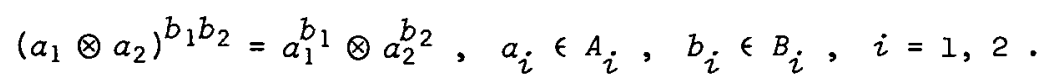

Notice that if $G$ is a non-nilpotent critical group in $\mathrm{V}$ then, using the notation of $\$ 4, G^{\#} \cong \mathrm{F} \# \mathrm{D}$ where $\mathrm{F}=\left(M_{1} H, M_{1}, H\right)$ and $\mathrm{D}=(N K, N, K)$; tensoring over $R_{\alpha}$ instead of $Z$ makes no difference since $M_{1} \otimes_{Z} N \cong M_{1} \otimes_{R_{\alpha}} N$ as $H K$-modules. If $S$ is a variety of bigroups denote by $S^{D}$ the variety of groups generated by

$$
\{F \# D: F \in S\} \text {. }
$$

We record without proof the following facts:-

$F_{1} \leq F$ implies $F_{1} \# D \leq F \# D$;

if $\zeta$ is a homomorphism of $F$ then the natural homomorphisms $B_{1} \times B_{2} \rightarrow B_{1} \zeta \times B_{2}$ and $A_{1} \otimes_{2} \lambda_{2} \rightarrow A_{1} \zeta \otimes_{2} A_{2}$ extend to $a$ homomorphism $F \# D+F \zeta \# D$;

$\left(\prod_{i} F_{i}\right) \# \mathrm{D}$ is a subdirect product of $\prod_{i} F_{i} \# \mathrm{D}$.

The next lenma follows easily from these three.

LEMMA 5.4. If $S$ is generated by the set $\left\{\mathrm{F}_{i}: i \in I\right\}$ then $S^{D}$ is generated by $\left\{F_{i} \# D: i \in I\right\}$.

COROLLARY 5.5. $\left(S_{1} \vee S_{2}\right)^{D}=S_{1}^{D} \vee S_{2}^{D}$.

The join irreducibles in $\Lambda(\underset{p}{(A)} \underset{q}{A} p)$ are either locally nilpotent or not; the former are described in [16] and the latter will be described in terms of join irredicuble subvarieties of the variety of bigroups $\stackrel{\underline{A}}{p}^{\alpha} \stackrel{\circ}{\stackrel{A}{=} p}$ (for which see $(4.3 .15$ ) of [5]) and irreducible linear groups in $\stackrel{A}{\Rightarrow} \stackrel{A}{q}:$

THEOREM 5.6. The non-locally nilpotent join-irreducible

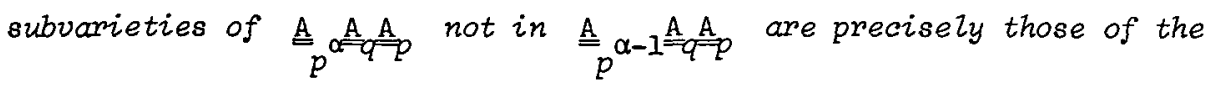
form $S^{D}$ where $S$ is a join-irreducible subvariety of $\stackrel{A}{p}_{p}^{\alpha} \stackrel{A}{\Rightarrow} p$ not in 
$\underline{\underline{E}} \circ \underline{A_{p}}$ and $\mathrm{D}=\left(D, A_{2}, B_{2}\right)$ has $A_{2}$ of exponent $p^{\alpha}$ and $B_{2} \in \stackrel{A_{A}}{\Rightarrow} p$ acting faithfully and irreducibly on $A_{2} / p A_{2}$.

Proof of Theorem 5.3. This result is covered by a more general (unpublished) result of J.M. Brady: a soluble just non-Cross variety of finite exponent, which is not $A_{p} A p$ or $\underset{P}{A} A A_{p}$, is contained in $\stackrel{\mathrm{A}}{\Rightarrow}\left(\underline{\mathbb{N}}_{c} \wedge \stackrel{\mathrm{B}}{=}\right) \quad(p \not n)$. We include a proof of Theorem 5.3 since it comes easily; it suffices to show that every subvariety $\underline{\underline{U}}$ of $\underline{\underline{V}}$ not containing $\stackrel{A}{\Rightarrow} A p$ is Cross. By Theorem 5 of Kovács and Newman [16], p-groups in $\underline{\underline{U}}$ have bounded class; and, in particular, nilpotent critical groups have bounded order (51.35 in [18]). If $G$ is a non-nilpotent critical group in $\underline{\underline{U}}$ then 51.38 in [18] ensures that $H$ has bounded order (using the notation of 54 ). If $T$ is the sylow $p$-subgroup of $K$ then $(\sigma G)_{T}$ is a direct sum of regular representations of $T$, by Theorem 2.2 and 65.16 in [8], and therefore $\operatorname{var} G$ contains a group, isomorphic to $C_{p}$ wrT , whose class is at least $|T|$. Hence $|T|$ is bounded, and Lemma 2.7 then shows that $|K|$ is bounded. Consequently Theorem 4.3 ensures that $|G|$ is bounded, thus showing that $\underline{\underline{U}}$ contains but finitely many critical groups and is Cross.

Proof of Theorem 5.2. If $\underline{W}_{i} \quad(i=1,2,3)$ are subvarieties of $\underline{\underline{V}}$ we need to show that

(5.7) $\quad \underline{\underline{W}}_{1} \wedge\left(\underline{\underline{W}}_{2} \vee \underline{\underline{W}}_{3}\right) \leq\left(\underline{\underline{W}}_{1} \wedge \underline{\underline{W}}_{2}\right) \vee\left(\underline{\underline{W}}_{1} \wedge \underline{\underline{W}}_{3}\right)$,

since the other inclusion is obvious. Let $G$ be a critical group belonging to the left-hand side of (5.7). By (1.12) and (1.14) of Kovács and Newman [14] there exist subsets $\Sigma_{i} \subseteq \underline{W}_{-}(i=1,2,3)$ of critical groups whose monoliths are similar to $\sigma G$ such that

$$
G \in \operatorname{var} \Sigma_{1} \wedge\left(\operatorname{var} \Sigma_{2} \vee \operatorname{var} \Sigma_{3}\right) ;
$$

and if $\Phi_{i}=\left\{F(X): X \in \Sigma_{i}\right\} \quad(i=1,2,3)$ (where $F(X)$ is the Fitting subgroup of $X$ ) then, using (1.14) in [14],

$$
F(G) \in \operatorname{var} \Phi_{1} \wedge\left(\operatorname{var} \Phi_{2} \vee \operatorname{var} \Phi_{3}\right)
$$

of course if $G$ is nilpotent then the members of each $\Sigma_{i}$ are nilpotent 
and, by Theorem 4 in [16],

$$
G \in\left(\operatorname{var} \Sigma_{1} \wedge \operatorname{var} \Sigma_{2}\right) \vee\left(\operatorname{var} \Sigma_{1} \vee \operatorname{var} \Sigma_{3}\right) \leq\left(\underline{\underline{W}}_{1} \wedge \underline{\underline{W}}_{2}\right) \vee\left(\underline{\underline{W}}_{1} \wedge \underline{\underline{W}}_{3}\right)
$$

as required; thus assume that $G$ is not nilpotent. Put

$F=\left(M_{1} H, M_{1}, H\right)$ in the notation of $\$ 4$ and then it is clear that $F, F(G)$

generate the same variety (indeed the bigroups they carry generate the same variety - cf. $(3.1 .6)$ in $[5])$; also write $F_{X}$ for the analogous bigroup corresponding to $X \in \Sigma_{i} \quad(i=1,2,3)$; and write $D=(N K, N, K)$. Hence, if

$$
\Psi_{i}=\left\{F_{X}: X \in \Sigma_{i}\right\}, i=1,2,3,
$$

then it follows from $(4.3 .4)$ in [5] and (5.8) that

$$
F \in \operatorname{var} \Psi_{1} \wedge\left(\operatorname{var} \Psi_{2} \vee \operatorname{var} \Psi_{3}\right)
$$

But by $(4.3 .14)$ in [5], $\Lambda\left(\underline{p}_{p}^{\alpha}{ }_{\underline{A}}^{A}\right)$ is distributive and therefore

$$
F \in\left(\operatorname{var} \Psi_{1} \wedge \operatorname{var} \Psi_{2}\right) \vee\left(\operatorname{var} \Psi_{1} \wedge \operatorname{var} \Psi_{3}\right) \text {. }
$$

However Corollary 5.5 shows that

$$
\begin{aligned}
G^{\#} \cong \mathrm{F} \# D \in\left(\operatorname{var} \Psi_{1} \wedge \operatorname{var} \Psi_{2}\right)^{D} \vee\left(\operatorname{var} \Psi_{1} \wedge \operatorname{var} \Psi_{3}\right)^{D} \\
\quad \leq\left(\left(\operatorname{var} \Psi_{1}\right)^{D} \wedge\left(\operatorname{var} \Psi_{2}\right)^{D}\right) \vee\left(\left(\operatorname{var} \Psi_{1}\right)^{D} \wedge\left(\operatorname{var} \Psi_{3}\right)^{D}\right) ;
\end{aligned}
$$

and $\left(\operatorname{var} \Psi_{i}\right)^{D} \leq \underline{W}_{i}$ by Lemma 5.4. Finally, then,

$$
G \in\left(\underline{\underline{W}}_{1} \wedge \underline{\underline{W}}_{2}\right) \vee\left(\underline{\underline{W}}_{1} \wedge \underline{\underline{W}}_{3}\right) .
$$

Proof of Theorem 5.1. Since $\underline{\underline{V}}$ is finitely based (two applications of Theorem 3.1 of Higman [11] show this) it suffices to prove that $\Lambda(\underline{\underline{v}})$ has descending chain condition. The following, easily proved, lemma will be used.

LEMMA 5.9. A Zocally finite variety $\underline{\underline{\mathrm{x}}}$ has descending chain condition on subvarieties if and only if to every set $\left\{G_{i}: i \in I^{+}\right\}$of non-isomorphic critical groups in $\underline{\underline{x}}$ there exists $i \in I^{+}$such that $(5.10)$

$$
G_{i} \in \operatorname{var}\left\{G_{j}: j \geq i+1\right\} \text {. }
$$

It will be convenient to restate here some facts from earlier 
sections, in the process establishing some notation.

(5.11). If $G \in \underline{\mathrm{V}}$ is critical and not nilpotent the Fitting subgroup $F(=A H)$ of $G$ has a complement $K$ (see $\$ 4$ ).

(5.12). $K=S T$ where $S \in \frac{A}{A}$ is the centralizer in $K$ of $\sigma K$ and $T \in \underline{A}$ P is a complement for $S$ in $K$.

(5.13). By Lema $2.7 S$ is a direct product of homocyclic subgroups $S_{i j}\left(1 \leq j \leq r_{i}, 1 \leq i \leq x\right)$ which are normal in $K$ and, as normal subgroups of $K$, indecomposable. (Assume that $\exp _{i j}=\exp S_{k l}$ if and only if $i=k$.$) The \sigma S_{i j}$ are precisely all the minimal normal subgroups of $K$ and $\sigma K$ is their direct product. Put $s_{i}=\prod\left(s_{i j}: 1 \leq j \leq r_{i}\right\} \quad(1 \leq i \leq r)$.

Now for some $\rho \in\{1, \ldots, r\}$ let $W$ be a normal subgroup of $K$ maximal with respect to containing $S_{1} S_{2} \ldots S_{\rho}$ and avoiding $S_{p+1} \ldots S_{r}$ (so that

$$
\left.W=S_{1} S_{2} \ldots s_{\rho} \cdot C_{T}\left(s_{\rho+1} \cdots s_{r}\right)\right) .
$$

For $i \in\{1, \ldots, \rho\}$ and arbitrary $j \in\left\{1, \ldots, r_{i}\right\}$ let $X_{i j}$ be a normal subgroup of $K$ maximal with respect to containing all $S_{k l}$ with $(k, i) \neq(i, j)$ and avoiding $s_{i j}$ (so that

$$
\left.x_{i j}=\left(\prod_{(k, Z) \neq(i, j)} S_{k \tau}\right) \cdot C_{T}\left(s_{i j}\right)\right) .
$$

Put

$$
\Gamma=K / W, \quad \Delta_{i j}=K / X_{i j}, \quad 1 \leq i \leq \rho, \quad 1 \leq j \leq r_{i} .
$$

LEMMA 5.14. Suppose $Z(K)=1$. Then $\Delta_{i j} \cong \Delta_{i 1}(1 \leq j \leq r)$ and if $\Sigma=\Gamma \times \prod_{i=1}^{p} \Delta_{i}^{r_{i}}$ (where $\Delta_{i}=\Delta_{i \perp}$ and $\Delta_{i}^{r_{i}}$ denotes the $r_{i}$-fold 
direct power of $\left.\Delta_{i}\right)$ there is an embedding $\mu: K \rightarrow \Sigma$ such that

$$
(\sigma K) \mu=\sigma \Sigma .
$$

Proof. Since $Z(K)=1$ none of $S_{i j}$ can be central, and each $\Delta_{i j}$ is isomorphic to $S_{i j}$ split-extended by an automorphism of order $p$. Consequently up to isomorphism $\Delta_{i j}$ is independent of $j$. By (5.13) $W \cap \bigcap_{i, j} X_{i j}=1$, the $\sigma S_{i j}$ being the only minimal normal subgroups of $K$. This completes the proof.

LEMMA 5.15. Suppose $Z(K)=1$. Put $C_{0}=C_{T}\left(S_{\rho+1} \ldots S_{p}\right)$ and $\left|T: C_{0}\right|=p^{\delta}$. Then provided $r_{i} \geq p^{\delta+s \rho}(1 \leq i \leq \rho), K$ contains $a$ subgroup $\Sigma_{0} \cong \Gamma \times \prod_{i=1}^{p} \Delta_{i}^{s}$ with $\sigma \Sigma_{0} \leq \sigma K$.

Proof. Note first that the number of inequivalent non-trivial irreducible representations of an elementary abelian group of order $p^{u}$ is at most $p^{u}-1$. Hence, since the minimal normal subgroups $\sigma S_{i j}$ of $K$ afford inequivalent representations of $T$, by (5.13), a subgroup of index $p^{u}$ in $T$ centralizes at most $p^{u}-1$ of the $\sigma S_{i j}$ and therefore centralizes at most $\left(p^{u}-1\right) S_{i j}{ }^{\prime}$. The proof depends on repeated use of this fact.

Since $r_{1} \geq p^{\delta+s p}>p^{\delta}-1$ there exists $j(1,1) \in\left\{1, \ldots, r_{1}\right\}$ such that

$$
C_{1}=C_{T}\left(S_{1 j(1,1)}\right) \neq C_{0} .
$$

But $\left|T: C_{1}\right|=p$ so that $\left|T: C_{0} \cap C_{1}\right|=p^{\delta+1}$. Suppose inductively that for some $\xi$, $\eta$ with $I \leq \xi \leq \rho$ and $0 \leq n<s$ we have chosen subgroups $C_{v}$ (of index $p$ ) of $T$, with $1 \leq v \leq(\xi-1) s+\eta$ such that

$$
\begin{aligned}
& \text { i) if } v=(\lambda-1) s+\mu \quad(1 \leq \lambda \leq \xi, 0 \leq \mu \leq n) \text { then } \\
& C_{v}=C_{T}\left(s_{\lambda j(\lambda, \mu)}\right) \text { for some } j(\lambda, \mu) \in\left\{1, \ldots, r_{\lambda}\right\} \text {; }
\end{aligned}
$$


ii) if $D_{v}=\bigcap_{w=0}^{v} C_{w}$ then $\left|T: D_{v}\right|=p^{\delta+v}$.

For $\xi=1, n=1$ we have done this.

Now $r_{\xi} \geq p^{\delta+s \rho}=p^{[\delta+(\xi-1) s+\eta]+[(\rho-\xi+1) s-\eta]}>p^{\delta+(\xi-1) s+\eta}-1$. Hence there exists $j(\xi, n+l) \in\left\{l, \ldots, r_{\xi}\right\}$ such that

$$
c_{(\xi-1) s+\eta+1}=C_{T}\left(S_{\xi_{j}(\xi, \eta+1)}\right) \neq D_{(\xi-1) s+n} .
$$

Also $C_{(\xi-1) s+n+1}$ has index $p$ in $T$ and therefore

$$
\left|T: D_{(\xi-1)_{s+n+1}}\right|=p^{\delta+(\xi-1) s+n+1}
$$

as required. In case $\xi<\rho$ and $\eta=s-1$ the proof of the inductive step is similar - we choose the next $C$ from among the centralizers of the $S_{\xi+1 j}$.

It may happen that $D_{\rho \mathcal{S}} \neq 1$. If that is the case continue choosing centralizers $C_{v}$, for $v>\rho s$, so that none contains the intersection of all previous $C^{\prime} s$ : this can be done since the intersection of the centralizers of all $S_{i j}$ is 1 (by (5.13)). Indeed if $\left|C_{0}\right|=p^{\gamma}$ then $\gamma$ is the first value of $v$ for which $D_{v}=1$. Put

$$
I_{v}=\bigcap_{w \neq v} C_{w}
$$

It is a simple matter to compute that

$$
I_{0} \text { is a complement for } C_{0} \text { in } T \text {; }
$$

and

for $v>0$, each $I_{v}$ has order $p$ and $C_{0}$ is their direct product.

Hence if

$$
\Gamma^{*}=I_{o} S_{\rho+1} \ldots S_{r}, \quad \Delta_{\xi \eta}^{*}=I_{v} S_{\xi j}(\xi, \eta), I \leq v=\xi s+\eta,
$$

then clearly $\Gamma^{*}, \Delta_{\xi}^{*}$ generate their direct product and, since $\Gamma^{*} \cong \Gamma$, 
$\Delta_{\xi n}^{*} \cong \Delta_{\xi}$, the proof of Lemma 5.15 is complete.

Suppose now that $S=\left\{G_{j}: j \in I^{+}\right\}$is a set of non-isomorphic critical groups in $\underline{\underline{V}}$. If infinitely many of them are nilpotent then (5.10) is satisfied for $S$ by Theorem 4 in [16]; hence we may suppose (by taking an infinite subset of $S$ instead if necessary) that all groups in $S$ are non-nilpotent. Further we may suppose that, in the notation of (5.13), the quantities $r, \exp _{i}(1 \leq i \leq r)$ are independent of $G \in S$ (again by replacing $S$ by an infinite subset if necessary). Choose $\rho \in\{1, \ldots, r\}$ by

$$
\rho+1 \leq i \leq r \Leftrightarrow\left\{r_{i}: G \in S\right\} \text { is bounded. }
$$

Then the groups $\Gamma$ corresponding to $G \in S$ have bounded order and hence we may suppose that $\Gamma$ is (up to isomorphism) independent of $G$. By (5.13) $Z(K)$ are all cyclic, hence there are but finitely many choices for $Z(K)$ and so we may assume that for $G \in S, Z(K)$ are all isomorphic. As a final simplification we may assume that the sequence of p-tuples $\left(r_{1}, \ldots, r_{\rho}\right)$ is ordered by components in the natural ordering of $S$.

With $S$ whittled down this far we have

LEMMA 5.16. For each $x \in I^{+}$there exists $j(x)>x$ and embeddings $\mu_{x z}: K_{x} \rightarrow K_{\eta}$ for $\tau \geq j(x)$ such that

$$
\left(\sigma K_{x}\right) \mu_{x l} \leq \sigma K_{Z}, \quad Z \geq j(x) \text {. }
$$

Proof. For $G \in S$ we may write

$$
K=Z(K) \times \hat{K}
$$

where $\hat{K}$ has trivial centre and satisfies the conditions (5.12), (5.13); and $Z(K)$ is cyclic, independent of $G$, and of course equal to some $S_{i j}$. By Lemmas 5.14 and 5.15 applied to the $\hat{K}^{\prime}$ 's if we choose $j=j(x)$ so that

$$
r_{i}\left(G_{j}\right) \geq p^{\delta+r_{i}\left(G_{x}\right) \rho}, \quad 1 \leq i \leq \rho,
$$

then there is a monomorphism $\mu_{x l}: \hat{K}_{x} \rightarrow \hat{K}_{l}(l \geq j(x))$, and this does 
what we want.

Finally consider the sequence in $I^{+}$defined by

$$
Z(1)=1, \quad Z(n)=j(Z(n-1)), n \in I^{+} \text {. }
$$

In the notation introduced at the beginning of this section if $G_{i} \in S$ then $G_{i} \cong F_{i} \# D_{i} \cdot$ By (4.2.29) in [5], and the analogue of Lemma 5.9 for bivarieties, there exists $n \in I^{+}$such that

$$
F_{Z(n)} \in \operatorname{svar}\left\{F_{Z(n+1)}, F_{Z(n+2)}, \ldots\right\} .
$$

Consequently there exists $i \quad(=Z(n))$ such that

$$
F_{i} \in \operatorname{svar}\left\{F_{Z(i)}, F_{Z(i)+1}, \ldots\right\}
$$

Now if $G \in\left\{G_{Z(i)}, G_{Z(i)+1}, \ldots\right\}$ Lemma 5.16 shows that $K$ has a subgroup $K^{*} \cong K_{i}$ with

$$
\sigma K^{*} \leq \sigma K
$$

Since $N$ is principal indecomposable, $N_{K^{*}}$ has a component $N^{*}$ which is principal indecomposable. By Lema $2.1 N^{*}$ is faithful for $K^{*}$ and by Theorem 2.2

$$
N^{*} K^{*} \cong N_{i} K_{i}
$$

Hence for each $G \in\left\{G_{Z}: l \geq j(i)\right\}, G^{\#}$ has a subgroup isomorphic to $F \# D_{i}$. It follows from $(5: 17)$ and Lemma 5.4 that $G_{i} \in \operatorname{var}\left\{G_{Z}: Z \geq j(i)\right\}$. By Lemma 5.9 the proof of Theorem 5.1 is complete.

Proof of Theorem 5.6. Suppose that $\underline{\underline{U}} \leq \underline{\underline{V}}$ is join-irreducible and not locally nilpotent. Since $\underline{\underline{U}}$ is generated by its critical groups it is generated by a set $S$ of non-nilpotent critical groups, and we may further assume that for all $G \in S$, expA is constant (in the notation of 54): we may as well assume that $\exp A$ are all equal to $p^{\alpha}$. We now show that the number of similarity classes of the $\sigma G(G \in S)$ is finite and therefore, of course, the groups of $S$ may be assumed to belong to a 
single similarity class. The next lemma accomplishes this.

LEMMA 5.18. Let $S^{\prime}$ be a set of non-nilpotent critical groups in $\underline{V}$ such that for all $G \in S^{\prime}, \exp A=p^{\alpha}$ and, furthermore, the sequence $\left\{|K|: G \in S^{\prime}\right\}$ is unbounded. Then varS' $=\underline{\underline{V}}$.

Proof. Let $G_{0}$ be an arbitrary critical group in $\underline{\underline{V}}$. We show that $G_{0}$ is in $\operatorname{var} G$ for some $G \in S^{*}$, this being sufficient to prove the lemma. It follows from Lemmas 5.14 and 5.15 (as in the proof of Lemma 5.16) that for all suitably large $K, K_{0}$ is isomorphic to a subgroup $\bar{K}$ of $K$ with

$$
\sigma \bar{K} \leq \sigma K
$$

Moreover since the index of $C_{T}(\bar{K})$ in $T$ is bounded in terms of $K_{0}$, we may assume that

$$
\left|C_{T}(\bar{K})\right| \geq\left|H_{0}\right|
$$

Put $\bar{H}=C_{T}(\bar{K})$. Then $N \overline{H K}$ is a direct sum of principal indecomposables of $\overline{H K}$ over $R_{\alpha}$ - call one $\bar{A}$ say. Now since $\bar{A}$ is monolithic and co-monolithic and since $\bar{K}$ acts faithfully and irreducibly on $\sigma \bar{A}$ (by (5.19) and Lemma 2.1) we can use $\$ 4$ to deduce that

$$
\bar{A} \cong \bar{M}_{1} \otimes_{R_{\alpha}} \bar{N}
$$

where $\bar{M}_{1}$ is a principal indecomposable of $\bar{H}$ and $\bar{N}$ a principal indecomposable of $\bar{K}$ (this follows since $\bar{M}_{1}$ is the regular of $\bar{H}$, hence $\sigma \bar{M}_{1}$ is one dimensional trivial and so $\sigma\left(\bar{M}_{1} \otimes N\right)_{\bar{K}} \cong \bar{N}$ whence kert $=0)$. If $\overline{\mathrm{F}}=(\overline{A H}, \bar{A}, \bar{H})$ and $\overline{\mathrm{D}}=(\overrightarrow{N K}, \bar{N}, \bar{K})$ then

$$
\overline{A H K} \cong \overline{\mathrm{F}} \# \overline{\mathrm{D}} \text {; }
$$

and since $F_{0}$ is clearly a homomorphic image of $\bar{F}, G_{0}^{f f}$ (and therefore $\left.G_{0}\right)$ is a homomorphic image of $\bar{F} \# \bar{D}$. This shows that $G_{0}$ is in $\operatorname{var} G$ for some $G \in S^{\prime}$ as required.

We may assume, therefore, that for some fixed $D=\left(D, A_{2}, B_{2}\right)$ with $A_{2}$ a faithful principal indecomposable $R_{\alpha} B_{2}$-module and $B_{2} \in \stackrel{A}{A} p$, 


$$
G \in S \Rightarrow G^{\#} \cong \mathrm{F} \# \mathrm{D}, \exists F \in \underset{\underline{p}^{\alpha}}{\alpha} \stackrel{\circ \underline{A}}{\underline{p}} .
$$

Hence by Lemma 5.4 if $S=\operatorname{svar}\{F: G \in S\}$,

$$
\mathrm{U}=S^{D} \text {. }
$$

By Corollary 5.5 and (1.14) of [14], $S$ is join irreducible.

Conversely if $S$ is join irreducible suppose

$$
S^{D}=\underline{\underline{U}}_{1} \vee \underline{\underline{U}}_{2}
$$

By (1.12) of [14] we may assume that each of $\underline{\underline{U}}_{1}, \underline{\underline{U}}_{2}$ is generated by critical groups the similarity class of whose monoliths is determined by $D$. That is we may assume $\underline{\underline{U}}_{1}, \underline{\underline{U}}_{2}$ generated by groups of the form $F$ \#

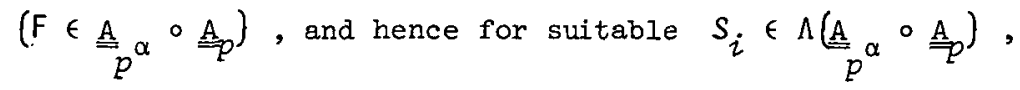

$$
\underline{\mathrm{U}}_{i}=s_{i}^{\mathrm{D}}, \quad i=1,2 \text {. }
$$

Corollary 5.5 and (1.14) of [14] then shows that $S=S_{1} \vee S_{2}$ whence $S_{1} \geq S_{2}$, say, and finally $\underline{\underline{U}}_{1} \geq \underline{\underline{U}}_{2}$. In other words $S^{D}$ is join-irreducible.

\section{References}

[1] J.M. Brady, R.A. Bryce and John Cossey, "On certain abelian-by-nilpotent varieties", BuzZ. Austral. Math. Soc. 1 (1969), 403-416.

[2] M.S. Brooks, "On varieties of metabelian groups of prime-power exponent", submitted to J. Austral. Math. Soc.

[3] Roger M. Bryant, "On some varieties of groups", BuzZ. London Math. Soc. 1 (1969), 60-64.

[4] R.A. Bryce, "Metabelian groups and varieties", Buzz. Austral. Math. Soc. 1 (1969), 15-25.

[5] R.A. Bryce, "Metabelian groups and varieties", Philos. Trans. Roy. Soc. London Ser. A 266 (1970), 281-355. 
[6] D.E. Cohen, "On the laws of a metabelian variety", J. Algebra 5 (1967), 267-273.

[7] P.J. Cossey, "On varieties of A-groups", Ph.D. Thesis, Australian National University, Canberra, 1966.

[8] Charles W. Curtis and Irving Reiner, Representation theory of finite groups and associative algebras (Interscience, New York, 1962).

[9] Marshall Hall, Jr, The theory of groups (Macmillan, New York, 1959).

[10] Graham Higman, "Complementation of abelian normal subgroups", Publ. Math. Debrecen 4 (1956), 455-458.

[11] Graham Higman, "The orders of relatively free groups", Proc. Internat. Conf. Theory of Groups, Austral. Nat. Univ., Canberra, 1965 (Gordon and Breach, New York, 1967).

[12] Graham Higman, "Some remarks on varieties of groups", Quart. $J$. Math. Oxford Ser. (2) 10 (1959), 165-178.

[13] Rudolf Kochendörffer, "Uber treue irreduzible Darstellungen endlicher Gruppen", Math. Nachr. 1 (1948), 25-39.

[14] L.G. Kovács and M.F. Newman, "On critical groups", J. Austraz. Math. Soc. $6(1966), 237-250$.

[15] L.G. Kovács and M.F. Newman, "Just-non-Cross varieties", Proc. Intermat. Conf. Theory of Groups, Austral. Nat. Univ., Canberra, 1965 (Gordon and Breach, New York, 1967).

[16] L.G. Kovács and M.F. Newman, "On non-Cross varieties of groups", $J$. Austral. Math. Soc. (to appear).

[17] R.C. Lyndon, "Two notes on nilpotent groups", Proc. Amer. Math. Soc. 3 (1952), 579-583.

[18] Hanna Neumann, Vaxieties of groups (Ergebnisse der Mathematik und ihrer Grenzgebiete, Band 37, Springer-Verlag, Berlin, Heidelberg, New York, 1967).

[19] Sheila Oates and M.B. Powell, "Identical relations in finite groups", J. Algebra I (1964), 11-39.

[20] D.R. Taunt, "On A-groups", Proc. Combridge Philos. Soc. 45 (1949), 24-42. 
[21] M.R. Vaughan-Lee, "Abelian by nilpotent varieties", Quart. J. Math. oxford Ser. (2) (to appear).

The Australian National University, Canberra, ACT. 